\title{
Turismo de Base Comunitária: proposta para o resgate cultural da comunidade quilombola Alto Itacuruçá, Abaetetuba (PA)
}

\section{Tourism Community-Based: a propose to the cultural rescue of the quilombola community Alto Itacuruçá, Abaetetuba (PA, Brazil)}

Dayara Vanessa de Souza Bezerra, Jonathan Rodrigues Nunes, Rayanne Silva Nascimento, Vânia Lúcia Quadros Nascimento

\section{RESUMO}

O Turismo de base comunitária tem se expandido pelo Brasil, devido ao incentivo à valorização da diversidade cultural, possibilitando aos municípios a exposição de suas atividades naturais e histórica assim como sua cultura tradicional, contribuindo diretamente na economia local. Neste sentido, este trabalho objetiva analisar 0 arcabouço cultural existente na comunidade Quilombola Alto Itacuruçá, ressaltando a inserção do turismo de base comunitária como uma alternativa para resgatar a memória cultural, haja vista a sua grande significância para a história do município de Abaetetuba (PA). Para tal se utilizará pesquisas bibliográficas e de campo, além de entrevistas com os moradores locais com intensão de compreender a etnicidade do espaço. Tais pesquisas subsidiarão a precursão da atividade turística na comunidade, atribuindo aos mesmos uma análise acadêmica da importância cultural que possuem, aliando a valorização e geração de opção de renda. Os resultados da pesquisa demonstram que a cultura tradicional é bastante expressiva no cotidiano das pessoas mais antigas da comunidade, as quais receberam o conhecimento passado por gerações, como brincadeiras tradicionais, cantigas, agricultura familiar, artesanatos e carpintaria. Entretanto houve uma interrupção nessa propagação da cultura para a juventude decorrente da modernização. O Turismo de base comunitária pode vir como um aliado para o enaltecimento dos saberes tradicionais, ao propor oficinas de qualificação, cujos instrutores serão os próprios patriarcas da comunidade, além de rodas de conversa resgatando a memória das raízes tradicionais, evidenciando a relevância e potencialidade atrativa que possuem para o mercado turístico.

PALAVRAS-CHAVE: Turismo de Base Comunitária; Comunidade Quilombola Alto Itacuruçá; Resgate da Memória Cultural. 


\section{ABSTRACT}

Tourism community-based enables more than inclusion of the autochthonous community, planning and management of tourism, the appreciation of cultural diversity, allowing municipalities the conscious use of its natural and historical attractions, as well as its traditional culture, contributing directly to the local economy. The aim of this study was to analyze the possibility of integration of community-based tourism in Quilombo Alto Itacuruçá (High Village Itacuruçá) community as an alternative to rescue the cultural memory, given its importance to the history of the city of Abaetetuba (PA, Brazil). Therefore, we conducted bibliographic and field research, with the information gathering tools and interviews with local residents. The results demonstrate that the local traditional culture is very expressive, being represented in the daily lives of older people in the community, which received the knowledge passed from generation to generation, which are cited as examples the traditional games, songs, family farming, crafts and carpentry. Also demonstrate that there was an interruption in this spread for today's youth as a result of the modernization. In conclusion, the communitybased tourism appears as an ally to the enhancement of traditional knowledge, proposing qualification workshops, whose instructors are the community patriarchs themselves, and conversation circles rescuing the local memory, highlighting the relevance and Attractive potential of the community for the tourist market.

KEYWORDS: Community-Based Tourism; Quilombo Alto Itacuruçá Community; Rescue of Cultural Memory.

\section{Introdução}

O Turismo de base comunitária (TBC) é um segmento do turismo que surgiu da necessidade trivalente: do mercado turístico de inovar, do estímulo à valorização cultural/ endógena e alternativa de renda para comunidades tradicionais. A comunidade autóctone é a grande protagonista dessas iniciativas e quando há planejamento estruturado pode proporcionar experiências mútuas.

De acordo com o Ministério do Turismo (2010), não existe conformidade em questão do conceito do TBC. O que ocorre devido a grande heterogeneidade de experiências, da especificidade do território e das distintas políticas adotadas pelas organizações não governamentais (ONG), responsáveis pela organização desses momentos. Entretanto, os conceitos definidos por estas possuem similaridades quanto à valorização do papel da comunidade e sua cultura, além da contribuição para a conservação dos recursos naturais, geração de renda e distribuição igualitária de seus resultados.

A Comunidade Remanescente de quilombo Alto Itacuruçá situa-se nas margens do rio Itacuruçá e faz parte do território quilombola. Titulado pelo Instituto de Terras do Pará (ITERPA) em junho de 2002, atualmente 
esse território é formado por mais sete comunidades além desta, no município de Abaetetuba (Pará)( Figura 1). A comunidade de Alto Itacuruçá é composta por 130 famílias, aproximadamente 4.000 moradores, divididos em setores no espaço de 12 mil hectares, e seu principal meio de subsistência consiste na agricultura familiar.

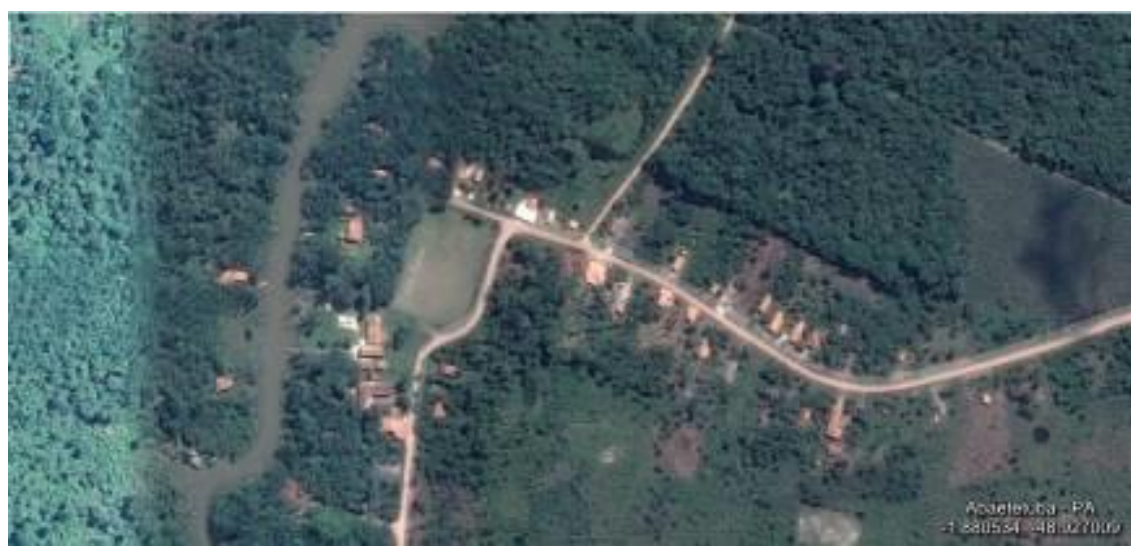

Figura 1: Localização da Comunidade remanescente de Quilombo Alto Itacuruçá. Figure 1:Location of the remaining Community Quilombo Alto Itacuruçá.

Fonte:Google maps (2016).

Source: Google maps (2016)

A cultura em uma comunidade quilombola quando pensada é remetida com excentricidade, algo significante e diferente do cotidiano de muitas pessoas, passada de geração a geração. Isto, para Maldonado (2009, p. 29), denomina-se patrimônio comunitário, pois é tudo aquilo que os distingue dos demais, como costumes, crenças, habilidades, artefatos, terras e manifestações tangíveis e intangíveis, ou seja, a expressão do seu "[...] modo de vida e organização social, sua identidade cultural e suas relações com a natureza". Portando, precisa ser, como tal, valorizado e propagado interna e externamente na comunidade.

Embora a cultura seja tão expressiva nessas comunidades, 0 desenvolvimento urbano e o tecnológico muitas vezes se tornaram prioridades no cotidiano da população. Ainda mais na faixa etária jovem, os responsáveis futuros pela perpetuação da cultura. Iniciou-se, então, o medo de que a história se perca no tempo, concomitantemente com o sentimento de impotência dos anciões, pensando que nada podem fazer.

O turismo de base comunitária vem à tona como uma alternativa de resgate dessa memória cultural. E a valorização da sua essência por toda a comunidade é um dever consigo e com toda a sociedade, tendo em vista que sua história contribuiu para a formação da sociedade miscigenada do Pará. Segundo Maldonado (2009, p. 29), "Diversas avaliações têm mostrado que, graças ao turismo, as comunidades estão cada vez mais conscientes do potencial de seus bens patrimoniais [...]", ou seja, buscam uma percepção mais ampla de sua cultura, pois sabem que precisam difundi-la aos turistas.

Bartholo (2014) afirma que essas relações comunitárias e suas singularidades podem ser fortalecidas por intermédio do turismo de base 
comunitária, se praticada de forma situada, priorizando as afirmações identitárias simbólicas do homo situs, não o homo economicus e suas pretensões universais. Além de fazer com que, a se pensar segundo Novo (2014), a comunidade tenha o sentimento de pertencimento e orgulho de sua história e grupo social a ponto de deixa-la conhecer por outros povos, acabando assim com o sentimento de inferioridade. Foi com o sentido de contribuir para isso que este trabalho foi realizado.

\section{Objetivo}

Analisar a possibilidade de inserção do turismo de base comunitária na comunidade Quilombola Alto Itacuruçá, como uma alternativa para resgatar a memória cultural.

\section{Metodologia}

Para a construção de uma análise concisa do tema proposto, foram realizadas pesquisas bibliográficas e de campo, tendo como instrumento de coleta de dados entrevistas semiestruturadas com os moradores locais com intensão de compreender a etnicidade do espaço. A pesquisa bibliográfica foi realizada sobre os temas turismo, turismo de base comunitária e comunidade quilombola; e a de campo foi realizada no período de 15 de fevereiro a 20 de março de 2016. Foram entrevistados 30 moradores, representando suas respectivas famílias.

\section{Resultados e Reflexões}

Segundo Loureiro (1989), "a cultura é a mais alta forma de expressão do homem e da sociedade. Ela é um instrumento através do qual se compreende o sistema social, garante-se a reprodução de seus valores, mas ao mesmo tempo, criam-se condições a transformação social". Tornando-se cada vez mais um potencial atrativo no mercado turístico.

$\mathrm{Na}$ Comunidade Remanescente de Quilombo Alto Itacuruçá percebeuse a riqueza cultural existente, algumas expressadas, ao ano, de forma contínua e outras esporádicas. Algumas dessas manifestações são as ladainhas antigas, reza cantada em latim pelos anciões da comunidade; as novenas, com duração de sete noites de rezas, em homenagem ao padroeiros de cada setor, encerrada com uma grande festa dançante; e o Círio de Nossa Senhora de Nazaré, padroeira da comunidade (Figura 2), que é realizado no mês de Setembro.

A Pajelança também possui um papel importante no local. De acordo com a moradora local e também curandeira Ana Ferreira, é um dom passado de geração a geração, nele o contemplado realiza rituais de curas com ervas medicinais, prenuncia acontecimentos e faz qualquer tipo de benzedura. Outros exemplos dos costumes da comunidade são o mutirão, que consiste na troca de mão de obra entre os vizinhos; além das profissões que também 
são passadas hereditariamente como a agricultura (Figura 3) e a fabricação de canoas artesanato em argila e tala.

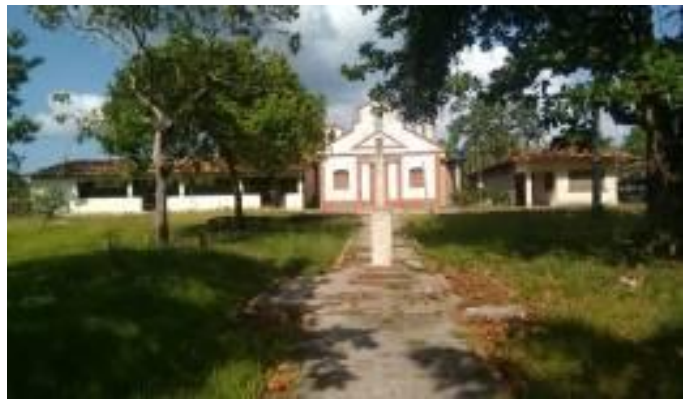

Figura 2: Capela Nossa Senhora de Nazareth. Figure 2: The Chapel of our lady of Nazareth Fonte: pesquisa de campo, 2016. Source: field research, 2016

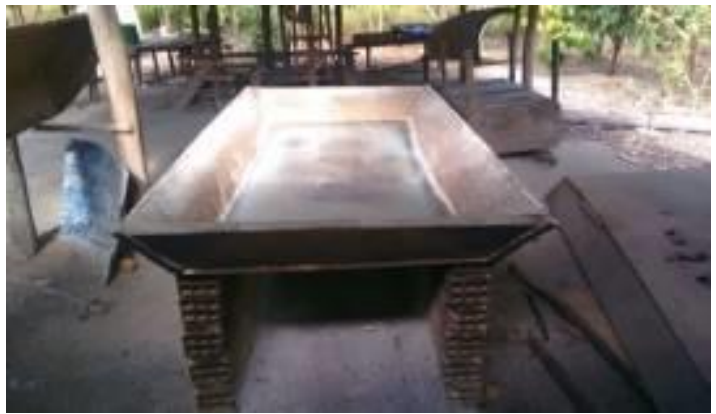

Figura 3: Retiro, para produção de farinha. Figure 3: Retiro (a house which flour is made), flour production.

Fonte: pesquisa de campo, 2016. Source: field research, 2016

Neste estudo constatou-se que, mesmo com grande simbologia, as tradições, valores e costumes da comunidade são mais valorizados pela população idosa, devido à proximidade ancestral com raízes. A cultura é passada de geração em geração. Entretanto, percebe-se a interrupção dessa propagação para os jovens por diversos fatores, algo que preocupa a comunidade como um todo, conforme demostrado no Quadro 1 abaixo.

Quadro 1: Percepção da valorização cultural pelos jovens.

Table 1: Perception of cultural appreciation by young people

\begin{tabular}{|c|c|c|c|c|c|c|}
\hline $\begin{array}{c}\text { Opinião } \\
\text { Faixa } \\
\text { Etária }\end{array}$ & Tecnologia & $\begin{array}{c}\text { Falta de } \\
\text { interesse }\end{array}$ & $\begin{array}{l}\text { Pouco } \\
\text { retorno } \\
\text { econômico }\end{array}$ & $\begin{array}{c}\text { Facilidade } \\
\text { de acesso } \\
\text { a outros } \\
\text { locais }\end{array}$ & $\begin{array}{l}\text { Acesso a } \\
\text { Informação }\end{array}$ & TOTAL \\
\hline $\begin{array}{l}\text { Menos } \\
\text { de } 30\end{array}$ & 8 & 2 & 1 & 7 & 3 & 21 \\
\hline 30 a 50 & 4 & 3 & 4 & 1 & 6 & 18 \\
\hline $\begin{array}{c}\text { Mais de } \\
50\end{array}$ & 5 & 5 & 3 & 2 & 2 & 17 \\
\hline TOTAL & 17 & 10 & 8 & 10 & 11 & 56 \\
\hline
\end{tabular}

Mesmo com conhecimento superficial sobre a atividade turística, $100 \%$ dos entrevistados acreditam que o turismo pode se tornar uma alternativa de estímulo à valorização da cultura para todas as faixas etárias da comunidade, bem como para melhorar a qualidade de vida, devido a investimentos em infraestrutura básica. Porém, o local não possui um conselho comunitário, o que torna deficiente e desunida qualquer iniciativa para melhora em grande escala. De maneira, unânime os entrevistados responderam que o turismo fomenta a necessidade dessa organização, 
ressaltando a importância de gestão da atividade assim como 0 esclarecimento de seus impactos negativos e positivos.

\section{Considerações}

O turismo de base comunitária contribui justamente para que essas comunidades tradicionais possam ver o quanto sua cultura é importante para sociedade, que a experiência e troca de conhecimento enriquece e ensina a humanidade a ser melhor. A comunidade remanescente de quilombo Alto Itacuruçá possui uma diversidade cultural notória, a qual precisa se manter viva no transcorrer dos anos, mesmo com todo o desenvolvimento urbano e suas imposições.

A atividade turística vem somar com as iniciativas de resgate da memória cultural, pois uma gestão responsável pode minimizar os impactos negativos e ampliar os benéficos, sempre com o enfoque que o turismo é uma alternativa atrelada ao meio de subsistência local. Iniciativas como o estabelecimento de um conselho comunitário são imprescindíveis à gestão, pois este, junto com as parcerias, poderá organizar oficinas de qualificação, com o fito de ensinar à juventude as profissões presentes na comunidade, assim como a forma mais eficiente de produção, dando-Ihes a oportunidade de ser instruído por seus próprios patriarcas. As rodas de conversa esclarecedoras sobre o turismo também poderiam ser mediadas pelo conselho, tendo em vista a representação das diretrizes da comunidade perante as parcerias. A valorização cultural é algo primeiramente interno, uma afirmação de identidade que refleti nas ações de um indivíduo, portanto não pode ser esquecida.

\section{Referências}

BARTHOLO, R. Sobre o lugar do Turismo de Base Comunitária In: NOVO, C.B.M.C.; CRUZ, J.G. (Orgs). Turismo Comunitário reflexões no contexto Amazônico. Manaus: Edua, 2014, pp. 41- 44.

BRASIL. Ministério de Turismo. Dinâmica e diversidade do turismo de base comunitária: desafio para a formulação de política pública. Brasília: Ministério do Turismo, 2010.

LOUREIRO, J.J.P. Estudos e problemas amazônicos: história social e econômica e temas especiais. Belém: Secretaria de Educação; Instituto do Desenvolvimento Econômico-Social do Pará, 1989.

MALDONADO, C. O turismo rural comunitário na América Latina: gênesis, características e políticas. In: BARTHOLO, R.; SANSOLO, D.G.; BURSZTYN, I. (Orgs). Turismo de Base Comunitária diversidade de olhares e experiências brasileiras. Rio de Janeiro: Letra e Imagem, 2009, p 25- 44.

NOVO, C.B.M.C. Turismo de Base Comunitária na região metropolitana de Manaus NOVO, C.B.M.C.; CRUZ, J.G. (Orgs). Turismo Comunitário reflexões no contexto Amazônico. Manaus: Edua, 2014, pp. 79- 102. 
Dayara Vanessa de Souza Bezerra: Universidade Federal do Pará, Belém, PA, Brasil.

E-Mail: dayarasouza22@gmail.com

Link para o currículo Lattes: http://lattes.cnpq.br/5376276452538445

Jonathan Rodrigues Nunes: Universidade Federal do Pará, Belém, PA, Brasil.

E-Mail: jonathanrodrigues58@hotmail.com

Link para o currículo Lattes: http://lattes.cnpq.br/4108256284598873

Rayanne Silva Nascimento: Universidade Federal do Pará, Belém, PA, Brasil.

E-Mail: nascimento_rayanne@hotmail.com

Link para o currículo Lattes: http://lattes.cnpq.br/9276306796576274

Vânia Lúcia Quadros Nascimento: Universidade Federal do Pará, Belém, PA, Brasil.

E-Mail: vluciaquadros@yahoo.com.brLink para o currículo Lattes: http://lattes.cnpq.br/1887894972158078

Data de submissão: 30 de agosto de 2016

Data de recebimento de correções: 31 de agosto de 2016

Data do aceite: 03 de outubro de 2016

Avaliado anonimamente 